\title{
Evaluation of Sweet Sorghum Juice for the Production of Lysine Using Corynebacterium glutamicum
}

\author{
David B. Johnston * (iD) and Nhuan Nghiem \\ Sustainable Biofuels and Coproducts Research Unit, Eastern Regional Research Center, USDA, \\ ARS. 600 East Mermaid Lane, Wyndmoor, PA 19038, USA; John.Nghiem@ars.usda.gov \\ * Correspondence: David.Johnston@ars.usda.gov; Tel.: +1-215-836-3756
}

Received: 14 March 2018; Accepted: 14 April 2018; Published: 19 April 2018

\begin{abstract}
Sweet sorghum juice is a liquid sugar feedstock that can be produced in non-tropical climates. Utilization of sweet sorghum juice as a feedstock for the production of lysine was investigated utilizing the auxotrophic mutant Corynebacterium glutamicum ATCC 21513 in stirred tank bioreactors. The juice was found to have inadequate nutrients for growth of the auxotroph alone and was supplemented with two levels of yeast extract and peptone. The supplemented juice could support growth and produce lysine from the available sugars. No inhibitory effects of the juice were found on the growth of the organism. The lysine concentrations reach $28.8 \mathrm{~g} / \mathrm{L}$ in the batch fermentations with complete utilization of the available sugars and a conversion efficiency of $0.23 \mathrm{~g} / \mathrm{g}$.
\end{abstract}

Keywords: lysine; sorghum; amino acids; bioconversion; Corynebacterium

\section{Introduction}

It is well established that the protein content and specific amino acid composition play a vital role in human and animal nutrition. Protein content and amino acid composition of animal feed diets also has a significant impact on growth rate, overall dietary protein need, and ultimately on environmental nitrogen release. Proper selection and formulation of the protein sources, in order to meet specific nutritional requirements, play a critical role in efficient and economic animal production.

Agricultural materials used in animal feed diets vary significantly in their nutritional content and value. Typically, feeds that are higher in protein concentration are more valuable; however, the amino acid profile also plays an important role in determining cost. Supplementation of animal diets with certain amino acids, such as L-lysine or L-threonine, has been shown to reduce the dietary crude protein requirements [1]. A low lysine level in a certain feed could reduce its value further, due to the need for additional supplementation to balance the amino acid profile. Corn and corn-based feeds fall into this category, as they have a relatively low L-lysine content and typically require blending or supplementation.

Ethanol production utilizing corn has greatly expanded the availability of distillers dried grains with solubles (DDGS). While the yeast component improves the nutritional value of the DDGS somewhat, the amino acid profile is still lacking in a number of essential amino acids including L-lysine [2].

L-lysine is a very important feed additive, as it is essential in animal diets. Chemical production of D,L-lysine has long been known; however, L-lysine production is currently done using microbial fermentations. Industrial production of L-lysine has been done using auxotrophic microbial mutants of Corynebacterium glutamicum since 1958 [3]. Currently, L-lysine production exceeds 2 million metric tons per year and is expected to reach almost 3 million by 2020 [4]. Other amino acids, such as 
L-glutamate, L-tryptophan and L-threonine, can also be produced utilizing different variants of Corynebacterium glutamicum [5].

Media composition and feedstock selection for production of biobased products by fermentation can have a substantial impact on the overall economics. Soluble sugar feedstocks can have advantages relative to conversion of starchy feedstocks such as corn or grain sorghum. Sugarcane, sweet sorghum and tropical maize are all potential sources for soluble sugar feedstocks for biobased products.

The utilization of sweet sorghum juice (SSJ) for the small-scale production of lysine by fermentation could potentially be used to upgrade animal feeds that have low lysine contents. Mixing the lysine containing fermentation broth with a nearly dry product such as corn or sorghum DDGS from ethanol production could result in an improved lysine DDGS product having substantially higher value.

Many different production strains for lysine production have been developed with different levels of auxotrophy and conversion efficiencies. Corynebacterium glutamicum ATCC 21513 is an auxotrophic mutant requiring L-homoserine, L-leucine, biotin, thiamin and pantothenic acid for growth [6]. The research reported here investigates the production of L-lysine using SSJ by fermentation using Corynebacterium glutamicum ATCC 21513. Yields are compared with defined media having equivalent sugar and nitrogen content to investigate any potential inhibitory effects of the SSJ.

\section{Materials and Methods}

\subsection{Materials}

The SSJ was produced during the 2015-growing season by Delta Biorenewables and was frozen immediately after pressing and coarse filtering. The juice was later thawed and centrifuged, and the supernatant filtered through Whatman GF/F glass fiber filter prior to use in fermentation media. Corynebacterium glutamicum ATCC 21513 was obtained from the American Type Culture Collection. The culture was maintained by streaking on nutrient agar and storage at $-80^{\circ} \mathrm{C}$ in glycerol. Lysine $(>98 \%)$, glucose, fructose, buffer salts and media components were obtained from Sigma-Aldrich (St. Louis, MO, USA). Amino acid reagents and standards were purchased from Agilent Technologies (Santa Clara, CA, USA). BD Bacto ${ }^{\mathrm{TM}}$ yeast extract and peptone were purchased from Fisher Scientific (Pittsburgh, PA, USA).

\subsection{Shake Flask Fermentations}

Shake flask fermentations were done in $125 \mathrm{~mL}$ Erlenmeyer flasks utilizing $25 \mathrm{~mL}$ of SSJ that had been either autoclaved or sterile filtered through $0.22 \mu$ filters. Juice was inoculated with $0.5 \mathrm{~mL}$ of an overnight liquid culture grown in nutrient broth that had been inoculated from a single colony grown on nutrient agar. Flasks were incubated at $30^{\circ} \mathrm{C}$ in a New Brunswick ${ }^{\mathrm{TM}}$ orbital shaking incubator at $250 \mathrm{rpm}$ with foam stoppers.

\subsection{Stirred Tank Bioreactor}

Fermentations were done using utilizing an Applikon Biotechnology controller with a 2-liter capacity vessel filled with 1 liter of SSJ or defined media. Air was sparged at 1 liter per min into the vessel and the dissolved oxygen levels maintained at $40 \%$ by cascading to speed control. The $\mathrm{pH}$ was maintained at 7.0 with the addition of $5 \mathrm{M}$ ammonium hydroxide.

\subsection{Media}

Media were prepared based on fermentation media described in [7]. SSJ was supplemented with the following per liter: $\mathrm{CaCl}_{2} \cdot 2 \mathrm{H}_{2} \mathrm{O}(1 \mathrm{~g}),\left(\mathrm{NH}_{4}\right)_{2} \mathrm{SO}_{4}(30 \mathrm{~g}), \mathrm{MgSO}_{4} \cdot 7 \mathrm{H}_{2} \mathrm{O}(0.4 \mathrm{~g}), \mathrm{NaCl}(0.05 \mathrm{~g})$, $\mathrm{MnSO}_{4} \cdot \mathrm{H}_{2} \mathrm{O}(0.0076 \mathrm{~g}), \mathrm{FeSO}_{4} \cdot 7 \mathrm{H}_{2} \mathrm{O}(0.001 \mathrm{~g}), \mathrm{KH}_{2} \mathrm{PO}_{4}(1 \mathrm{~g}), \mathrm{K}_{2} \mathrm{HPO}_{4}(1 \mathrm{~g})$, urea $(2 \mathrm{~g})$, yeast extract $(1.5 \mathrm{~g})$, peptone $(2 \mathrm{~g})$, thiamine $(0.2 \mathrm{mg})$, D-biotin $(0.5 \mathrm{mg})$, and L-serine $(0.1 \mathrm{mg})$. The defined media were prepared in deionized (DI) water instead of SSJ, and D-glucose $(70 \mathrm{~g})$ and D-fructose $(50 \mathrm{~g})$ were 
added, in addition to the supplements used with SSJ. The ammonium sulfate was prepared at $10 \times$ concentration in $100 \mathrm{~mL}$ of DI water and sterilized separately. It was then added to the bioreactor after autoclaving.

A richer growth medium with higher levels of yeast extract and peptone was also used with the SSJ as well as with the defined media. This medium utilized the same levels as above but the yeast extract and peptone contents were increased to $20 \mathrm{~g} / \mathrm{L}$ and $4 \mathrm{~g} / \mathrm{L}$, respectively.

\subsection{Inoculum Preparation}

Inoculum for the bioreactors was prepared in two $250 \mathrm{~mL}$ Erlenmeyer flasks with $50 \mathrm{~mL}$ of BD Difco ${ }^{\mathrm{TM}}$ nutrient broth using a single colony from a petri plate culture. Flasks were incubated at $30{ }^{\circ} \mathrm{C}$ in a New Brunswick ${ }^{\mathrm{TM}}$ orbital shaking incubator at $250 \mathrm{rpm}$ for $48 \mathrm{~h}$.

\subsection{HPLC Analysis for Lysine and Sugars}

Sugars (glucose and fructose), organic acids (lactic, succinic and acetic acid) and amino acids were quantitated using HPLC analysis as previously described in Johnston and McAloon [8]. Samples for lysine quantitation were diluted in DI water as necessary to be within the calibrated range (below $0.25 \mathrm{~g} / \mathrm{L}$ ). Duplicate injections were made for all samples.

\subsection{Biomass Determination}

Culture biomass contents were determined using $5 \mathrm{~mL}$ samples of culture media. The media were centrifuged, and the supernatant removed by aspiration. The pellet was washed one time with $5 \mathrm{~mL}$ of DI water and then transferred to a tared weigh boat and dried at $135^{\circ} \mathrm{C}$ for $2 \mathrm{~h}$ to determine dry weight. Determinations were done in duplicate and results averaged.

\subsection{Statistical Analysis}

Fermentations in shake flasks and bioreactors were done in duplicate, and chemical analyses were performed at least in duplicate. A $t$-test was used to determine statistical significance between treatments at $p \leq 0.05$. Mean and standard deviations are reported.

\section{Results and Discussion}

\subsection{Shake Flask Cultivation for Lysine Biosynthesis}

Initial experiments for lysine production were done in shake flasks. Table 1 shows the cell mass, lysine produced, sugars and final $\mathrm{pH}$ of the flasks. Initial $\mathrm{pH}$ of the SSJ was adjusted to $\mathrm{pH} 6.5$ and the final $\mathrm{pH}$ was measured to determine if there were changes. Using non-supplemented SSJ demonstrated that the juice alone was inadequate to support any significant growth of the organism. Corynebacterium glutamicum ATCC 21513 is an auxotrophic mutant requiring L-homoserine, L-leucine, biotin, thiamin and pantothenic acid for growth [6]. Supplementation with either urea or ammonium sulfate to alleviate the limited nitrogen content of the SSJ did allow growth and minimal lysine production. The growth and sugar utilization further indicated that additional supplementation was necessary.

Addition of yeast extract, which contains some quantity of the required nutrients, at 3 and $5 \mathrm{~g} / \mathrm{L}$, in addition to the nitrogen supplementation, improved growth and lysine production significantly. The level of production of organic acids, such as lactic acid, by the organism was so high that it was no longer possible to adequately maintain the $\mathrm{pH}$ within acceptable conditions for complete utilization of sugars in the SSJ (approximately 12\% $w / v$ ).

The information developed from the shake flask experiments clearly demonstrated that it was necessary to better maintain the $\mathrm{pH}$ for optimal lysine production. Addition of a phosphate buffer helped but still did not allow complete sugar utilization either. Utilization of a $\mathrm{pH}$-controlled 
bioreactor would therefore be necessary in order to better study the use of sweet sorghum juice for lysine production.

Table 1. Post fermentation media analysis after $72 \mathrm{~h}$ of incubation ${ }^{\mathrm{a}}$.

\begin{tabular}{|c|c|c|c|c|c|}
\hline Component $^{b}$ & Biomass & Lysine $^{c}$ & Glucose & Fructose & End $\mathrm{pH}^{\mathrm{c}}$ \\
\hline \multicolumn{6}{|c|}{$\mathrm{g} / \mathrm{L}$} \\
\hline No Addition & 0 & 0 & $90.5 \pm 3.6$ & $62.0 \pm 2.6$ & 6.5 \\
\hline YE $3 \mathrm{~g} / \mathrm{L}$ & $2.13 \pm 0.06$ & 0.27 & $77.5 \pm 0.3$ & $58.6 \pm 0.1$ & 6.81 \\
\hline YE $5 \mathrm{~g} / \mathrm{L}$ & $2.93 \pm 0.21$ & 0.42 & $67.8 \pm 0.8$ & $60.3 \pm 0.5$ & 6.05 \\
\hline YE + AMS-1 & $4.01 \pm 0.01$ & 2.81 & $66.0 \pm 0.8$ & $52.9 \pm 0.1$ & 4.95 \\
\hline YE + AMS-2 & $4.54 \pm 0.11$ & 2.43 & $47.6 \pm 0.3$ & $50.9 \pm 0.2$ & 6.28 \\
\hline YE + AMS-3 & $3.05 \pm 0.16$ & 1.92 & $50.3 \pm 0.3$ & $48.3 \pm 0.3$ & 9.03 \\
\hline $\mathrm{YE}+\mathrm{AMS}-4$ & $2.99 \pm 0.04$ & 1.31 & $60.0 \pm 0.3$ & $49.8 \pm 0.2$ & 9.94 \\
\hline YE + AMS-5 & $2.84 \pm 0.17$ & 0.52 & $63.1 \pm 0.9$ & $48.7 \pm 0.1$ & 10.0 \\
\hline YE + Urea-1 & $4.04 \pm 0.06$ & 2.65 & $65.3 \pm 0.5$ & $52.8 \pm 0.1$ & 5.08 \\
\hline YE + Urea-2 & $4.67 \pm 0.01$ & 2.66 & $49.1 \pm 0.1$ & $51.1 \pm 0.1$ & 6.44 \\
\hline YE + Urea-3 & $3.16 \pm 0.04$ & 2.20 & $47.4 \pm 0.6$ & $49.0 \pm 0.2$ & 8.80 \\
\hline YE + Urea-4 & $2.79 \pm 0.01$ & 1.26 & $61.7 \pm 0.2$ & $51.1 \pm 0.0$ & 9.98 \\
\hline YE + Urea-5 & $2.64 \pm 0.03$ & 0.53 & $62.8 \pm 0.1$ & $48.7 \pm 0.1$ & 10.1 \\
\hline
\end{tabular}

a Data shown are the average determinations for each condition \pm one standard deviation. Glucose and fructose values shown are the residual levels; ${ }^{\mathrm{b}}$ Ammonium sulfate (AMS) is added at 2.5, 12.5, 25, 37.5 and $50 \mathrm{~g} / \mathrm{L}$ indicated 1 through 5 respectively. Urea is added at 5, 25, 50, 150 and $200 \mathrm{~g} / \mathrm{L}$ indicated as 1 though 5 respectively. Yeast extract (YE) was added at $3 \mathrm{~g} / \mathrm{L}$ except were indicated; ${ }^{\mathrm{c}}$ Lysine and $\mathrm{pH}$ shows average determinations only.

\subsection{Lysine Biosynthesis in Stirred Tank Bioreactors (Using Low Supplementation Medium)}

The defined media were made by utilizing the same components added to the SSJ, and additionally included glucose and fructose at concentrations similar to those in the SSJ. This was done in order to perform a comparison with the SSJ to determine if there were any inhibitory components present in the SSJ that may impact the growth or lysine production. SSJ is known to contain about $1 \%$ trans-aconitic acid [9]. The cis- form of aconitic acid is an intermediate in the conversion of citrate to isocitrate; however, the trans- form commonly accumulates in range grasses, including sorghum [10,11]. Trans aconitic acid has been shown to have a range of inhibitory activities from antinutritional and antifeedant, nematicidal and antileishmanial properties [10,12-15].

At the lower levels of yeast extract $(1.5 \mathrm{~g} / \mathrm{L})$ and peptone $(2.0 \mathrm{~g} / \mathrm{L})$ supplementation, the defined media and the supplemented SSJ both reached similar levels of lysine production. Figure 1 shows the biomass, lysine, glucose and fructose levels during the fermentation period. While growth did occur, the lysine levels were much lower than expected. The average lysine levels for the SSJ and defined media after $96 \mathrm{~h}$ were $5.8 \pm 0.06$ and $4.0 \pm 0.04 \mathrm{~g} / \mathrm{L}$, respectively. Additionally, there was still considerable unfermented sugar remaining in the bioreactor. Under these conditions, only about $25 \%$ of the total sugars available were utilized in the SSJ and in the defined media. These results were somewhat surprising, because shake flask experiments indicated that $\mathrm{pH}$ control was likely the problem with having incomplete sugar conversion, which should have resulted in increased lysine production. 


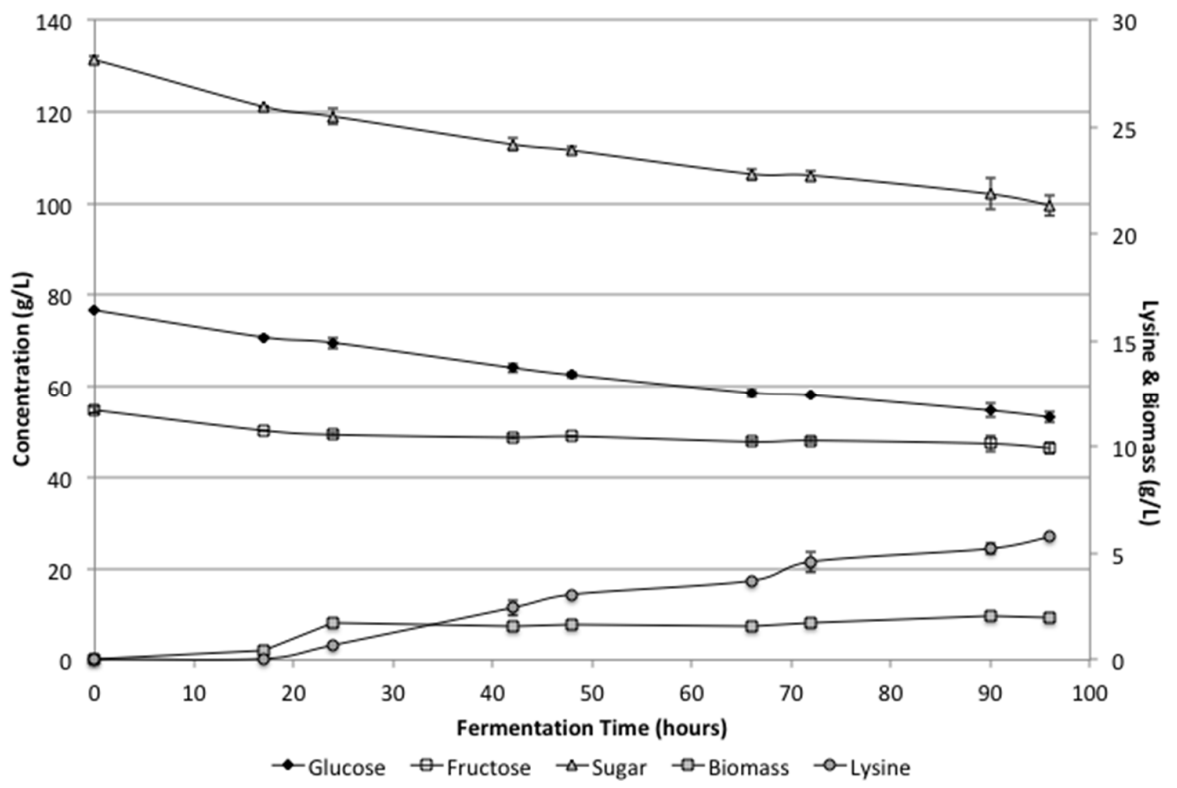

(A)

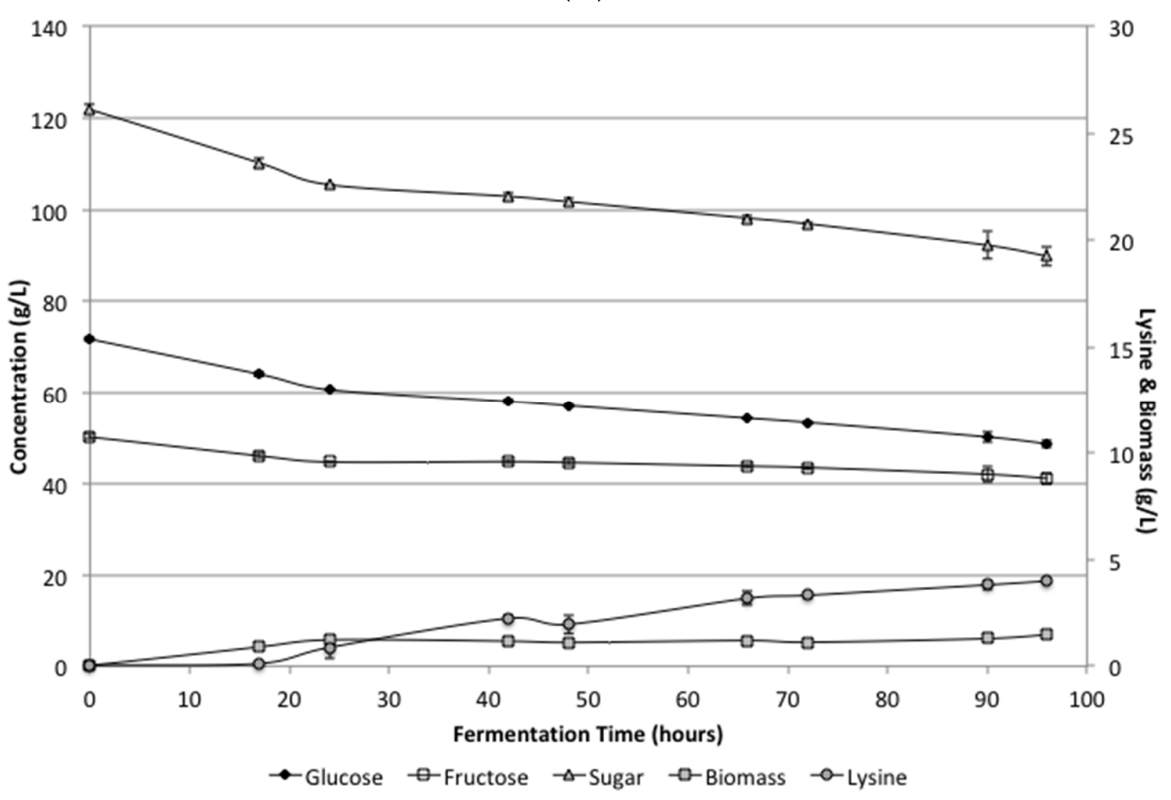

(B)

Figure 1. Sweet sorghum juice (A) and defined media (B) utilizing yeast extract (1.5 g/L) and peptone $(2.0 \mathrm{~g} / \mathrm{L})$. Data shown are the average values of duplicate fermentation. Error bars represent \pm one standard deviation of measured values.

Analysis of the biomass levels produced with the low supplementation indicated that there was still inadequate cell growth to complete the fermentation during the $96 \mathrm{~h}$ utilized. Additionally, a careful analysis of the rates further indicated that sugar was still being consumed and lysine was being produced at the same rates when the fermentation was stopped. The continued utilization was similar for both the defined media and the SSJ indicating that there was no apparent inhibition of the organism under these conditions.

\subsection{Lysine Biosynthesis in Stirred Tank Bioreactors (Using High Supplementation Medium)}

The results with the lower level of supplementation indicated that additional nutrients were necessary for adequate growth of the organism in SSJ. To address this issue, additional fermentations 
were done using higher levels of yeast extract and peptone, $20 \mathrm{~g} / \mathrm{L}$ and $4.9 \mathrm{~g} / \mathrm{L}$ respectively. It was likely that the levels of other added components were sufficient, and therefore, these were kept at the same levels as in the previous fermentation.

The defined media and SSJ fermentations again both showed nearly identical growth and conversion rates. Figure 2 shows the biomass, lysine, glucose and fructose levels during the fermentation period. Unlike the low supplementation fermentation, these results showed complete utilization of the sugars. Glucose was completely consumed by $48 \mathrm{~h}$. Fructose was utilized somewhat slower than glucose, but all sugars were consumed by $72 \mathrm{~h}$. Biomass levels were determined during the fermentation and reach $12 \mathrm{~g} / \mathrm{L}$ by $48 \mathrm{~h}$ in the defined media and $14 \mathrm{~g} / \mathrm{L}$ in the SSJ fermentations.

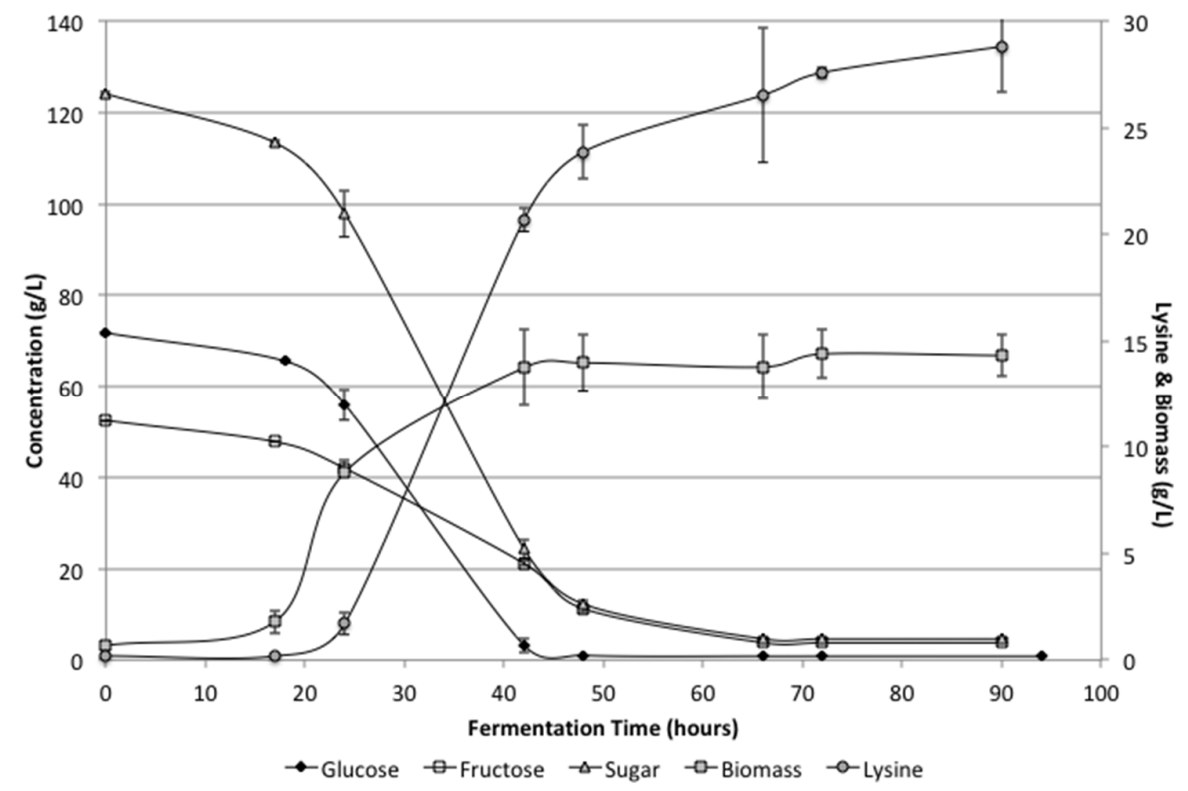

(A)

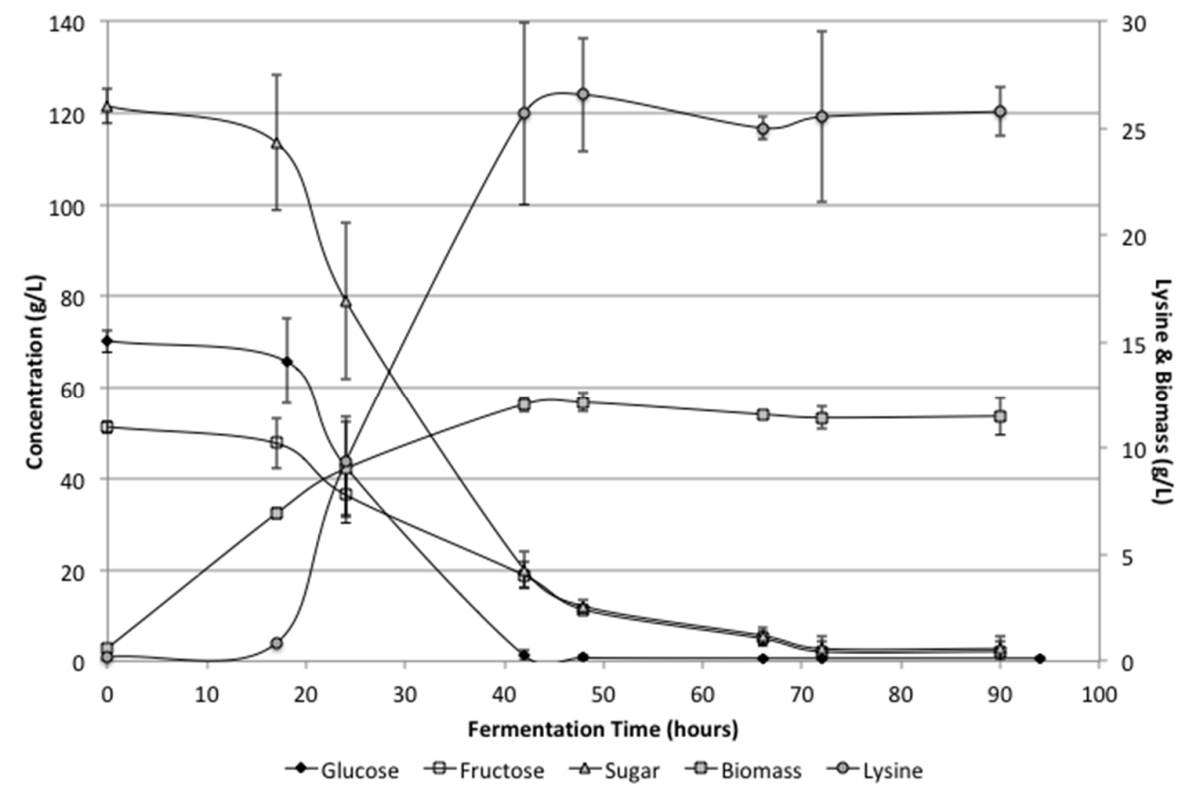

(B)

Figure 2. Sweet sorghum juice (A) and defined media (B) utilizing yeast extract ( $20 \mathrm{~g} / \mathrm{L})$ and peptone $(4.0 \mathrm{~g} / \mathrm{L})$. Data shown are the average values of duplicate fermentation. Error bars represent \pm one standard deviation of measured values. 
Lysine levels were increased significantly over the low supplementation results and resulted in final concentrations in the defined media and SSJ of $25.7 \pm 1.17$ and $28.8 \pm 2.14 \mathrm{~g} / \mathrm{L}$, respectively. There was a difference between the defined media and SSJ on average; however, it was not found to be statistically significant. The conversion efficiency for the defined media and SSJ were determined to be 0.21 and $0.23 \mathrm{~g}$ lysine/g sugar, respectively. Lysine concentrations and conversion efficiencies would likely be improved if a fed-batch system were to be utilized.

\section{Conclusions}

In conclusion, shake flask data showed that Corynebacterium glutamicum ATCC 21513 could be grown and produce lysine in SSJ. Growth in the juice was only possible when additional nitrogen, as well as other nutrients, were supplemented. Utilizing stirred tank bioreactors for improved control of $\mathrm{pH}$ successfully demonstrated that lysine could be produced utilizing SSJ as a carbon source. All of the sugars in the juice could be utilized under the bioreactor conditions when adequate growth occurred. Cell growth and lysine production were essentially identical in the defined media and SSJ, indicating that there was no inhibitory activity of the juice. Lysine yields were consistent with literature values based on availability of the carbon source. It is reasonably expected that increased concentrations of lysine could be produced utilizing SSJ in a fed-batch system.

Acknowledgments: The authors would like to thank Jennifer Thomas for her work in helping to conduct the fermentation experiments and for doing the sample analysis.

Author Contributions: David B. Johnston and Nhuan Nghiem conceived and designed the experiments; David B. Johnston performed the experiments and David B. Johnston and Nhuan Nghiem analyzed the data; Nhuan Nghiem contributed equipment/reagents; David B. Johnston wrote the paper and Nhuan Nghiem edited and reviewed.

Conflicts of Interest: The authors declare no conflict of interest. The work was funded by the United States Department of Agriculture, Agricultural Research Service and had no role in the design of the study; in the collection, analyses, or interpretation of data; in the writing of the manuscript, and in the decision to publish the results.

\section{References}

1. Huber, L.; de Lange, C.F.M.; Ernst, C.W.; Krogh, U.; Trottier, N.L. Impact of improving dietary amino acid balance for lactating sows on efficiency of dietary amino acid utilization and transcript abundance of genes encoding lysine transporters in mammary tissue. J. Anim. Sci. 2016, 94, 4654-4665. [CrossRef] [PubMed]

2. Liu, K.S. Chemical composition of distillers grains, a review. J. Agric. Food Chem. 2011, 59, 1508-1526. [CrossRef] [PubMed]

3. Nakayama, K.; Kinoshita, S.; Kitada, S. Studies on lysine fermentation. 1. Control mechanism on lysine accumulation by homoserine and threonine. J. Gen. Appl. Microbiol. 1961, 7, 145-154. [CrossRef]

4. Perez-Garcia, F.; Peters-Wendisch, P.; Wendisch, V.F. Engineering Corynebacterium glutamicum for fast production of L-lysine and L-pipecolic acid. Appl. Microbiol. Biot. 2016, 100, 8075-8090. [CrossRef] [PubMed]

5. Hirasawa, T.; Shimizu, H. Recent advances in amino acid production by microbial cells. Curr. Opin. Biotechnol. 2016, 42, 133-146. [CrossRef] [PubMed]

6. Sassi, A.H.; Coello, N.; Deschamps, A.M.; Lebeault, J.M. Effect of medium composition on L-Lysine production by a variant strain of Corynebacterium glutamicum Atcc-21513. Biotechnol. Lett. 1990, 12, 295-298. [CrossRef]

7. Razak, M.A.; Viswanath, B. Optimization of fermentation upstream parameters and immobilization of Corynebacterium glutamicum MH 20-22 B cells to enhance the production of L-lysine. 3 Biotech 2015, 5, 531-540. [CrossRef] [PubMed]

8. Johnston, D.B.; McAloon, A.J. Protease increases fermentation rate and ethanol yield in dry-grind ethanol production. Bioresour. Technol. 2014, 154, 18-25. [CrossRef] [PubMed]

9. Burau, R.; Stout, P.R. Trans-Aconitic acid in range grasses in early spring. Science 1965, 150, $766-767$. [CrossRef] [PubMed] 
10. Stout, P.R.; Brownell, J.; Burau, R.G. Occurrences of trans-aconitate in range forage species. Agron. J. 1967, 59, 21-24. [CrossRef]

11. Molloy, L.F. Determination of trans-aconitic acid in forage grasses. J. Sci. Food Agric. 1969, 20, $238-241$. [CrossRef]

12. Kim, M.; Koh, H.-S.; Obata, T.; Fukami, H.; Ishii, S. Isolation and identification of trans-aconitic acid as the antiffeedant in barnyard grass against the brown planthopper, Nilaparvata lugens (STAL) (Hompotara: Delphacidae). Appl. Entomol. Zool. 1976, 11, 53-57. [CrossRef]

13. Katsuhara, M.; Sakano, K.; Sato, M.; Kawakita, H.; Kawabe, S. Distribution and production of trans-aconitic acid in barnyard grass (Echinochloa crus-galli var. Oryzicola) as putative antifeedant against brown planthoppers. Plant Cell Physiol. 1993, 34, 251-254.

14. Du, C.Y.; Cao, S.Y.; Shi, X.Y.; Nie, X.T.; Zheng, J.S.; Deng, Y.; Ruan, L.F.; Peng, D.H.; Sun, M. Genetic and biochemical characterization of a gene operon for trans-aconitic acid, a novel nematicide from Bacillus thuringiensis. J. Biol. Chem. 2017, 292, 3517-3530. [CrossRef] [PubMed]

15. Kar, S.; Kar, K.; Bhattacharya, P.K.; Ghosh, D.K. Experimental visceral leishmaniasis—Role of trans-aconitic acid in combined chemotherapy. Antimicrob. Agents Chemother. 1993, 37, 2459-2465. [CrossRef] [PubMed]

(C) 2018 by the authors. Licensee MDPI, Basel, Switzerland. This article is an open access article distributed under the terms and conditions of the Creative Commons Attribution (CC BY) license (http:/ / creativecommons.org/licenses/by/4.0/). 